\title{
CRACKED BI-MATERIAL STRUCTURE SUBJECTED TO MONOTANICALLY INCREASING THERMAL LOADING. DETERMINATION OF THE INTERFACIAL SHEAR AND PEELING STRESSES
}

\author{
Gergana Nikolova, Jordanka Ivanova \\ Institute of Mechanics, Bulgarian Academy of Sciences, Sofia, Bulgaria \\ e-mail: gery@imbm.bas.bg; ivanova@imbm.bas.bg
}

\begin{abstract}
A modified analytical shear lag model is used for the evaluation of the interfacial shear and peeling stresses in a cracked bi-material structure composed of two elastic plates bonded together by an interface zero thickness material and subjected to monotonically increasing thermal loading. The "peeling" stress can be determined by the aid of the interfacial shear stress and is proportional to deflections of the thinner plate of the structure. The interface is assumed to exhibit brittle failure when the shear stress reaches the critical value. The analytical solution and the length of the debonding and intact zones as well as the interfacial shear and peeling stresses for given material properties and thermal loading are discussed and illustrated in figures.
\end{abstract}

Keywords: cracked bi-material plates, monotonic thermal loading, interfacial shear and peeling stresses

\section{Introduction}

Interfacial stresses are the main driving factors for the initiation of delamination. To minimize such failure in multi-layered structures, it is important to develop a better understanding of the stress distribution in the interface. The shear lag approach is one of the most used analytical tools in mechanics of composite materials. The papers of Dowling and Burgan (1990), Nairn (1988a,b) as well as the basic paper of Cox (1952) trace the interest in shear lag of aircraft and ship design from the early days to the more recent attention devoted to it by structural engineers. The shear lag model has been adopted and successfully used by different authors. In the papers of Nikolova et al. (2006), Nikolova (2008), Nikolova and Ivanova (2013), the shear lag approach is applied to a bi-material layered structure with the pre-cracked first layer. Different loadings are considered: static, thermal and combined thermo-mechanical ones.

The shear lag approach is also intensively used in the interface fracture mechanics considering the cracking, decohesion and delamination of the thin film on a substrate. A very important basic case in the fracture mechanics of thin films is the problem of a crack in the film oriented perpendicular to the film/substrate interface with the crack tip touching the interface and of a crack of the same geometry, but with length less than the film thickness, so that the crack tip is within the film. This problem was investigated by Beuth (1992) and Beuth and Klingbeil (1996). The plastic yielding of the substrate and film is accompanied by vertical cracking in the films and the interface fracture as well as delamination from the ends of the vertical crack in the film and the crack extension in the substrate along the interface (Hutchinson and Suo, 1991).

Interfacial shear and peeling stresses of layered composite materials under thermal loading were analysed in numerous papers. In most of them, the analysis is based on linear fracture mechanics assumptions when both element cracking and interface debonding are treated as mixed mode crack propagation with critical conditions expressed in terms of stress intensity factors, cf. Zhang (2000), Bleeck et al. (1998), Sorensen et al. (1998). 
The approximate analytical model for the assessment of interfacial stresses in a bi-material soldered assembly with a low-yield-stress of the bonding material was presented by Suhir (2006).

The aim of this paper is to investigate the interfacial shear and peeling stresses at the interface of a cracked bi-material structure subjected to monotonically increasing thermal loading. An approximate predictive model (Nikolova et al., 2006; Nikolova, 2008; Nikolova and Ivanova, 2013) is developed for the evaluation of interfacial thermal stresses in a bi-material structure. This material is considered linearly elastic at the stress level below the critical point and ideally plastic at higher stresses.

The delamination process is analysed for a cracked bi-material structure with stress free boundary. The plates are assumed as linear elastic and isotropic with different stiffness and thermal expansion moduli. The analytic solutions for the shear and peeling stresses are obtained. The main objective of the paper is to discuss the effect of the interface parameters and interfacial stresses on the delamination process.

\section{Assumptions and problem formulation}

\subsection{Assumption}

The following major assumptions are made in our analysis:

- The approximate analytical model can be used to present the influence of the temperature on behavior of the peeling stress and length of the interface debonding zone in the pre-cracked two plates structure with an interface zero thickness adhesive layer and subjected to thermal loading.

- At least one of the structure components (the "plate $A$ " /"plate $B$ ") is thick and stiff enough, so that this structural component and the construction as a whole do not experience bending deformations.

- The bonding material behaves linearly in the elastic stage. When the induced shearing stress exceeds the critical value, the interface debonding occurs.

- The interfacial stresses can be evaluated based on the concept of the interfacial compliance without considering the effect of "peeling". The "peeling" stress can be then determined from the evaluated interfacial shear stress. Due to this assumption, the "peeling" stress is proportional to the deflections of the thinner plate of the assembly, i.e., to its displacements with respect to the thicker plate.

\subsection{Two-plate structure model and basic equations}

Let us consider two elastic plates $A$ and $B$ (the plate $A$ has a normal [transverse] crack to the interface). The plates have different material properties and thermal expansion coefficients $\alpha_{A}, \alpha_{B}$ and they are bonded along the interface $I$ and loaded by a monotonically increasing thermal loading $\Delta T$ (see Fig. 1).

The modified shear lag model is applied and the plate bending is neglected, according to the second assumption (see Section 2.1). The interface is supposed to be with negligible thickness and works only on shear (Nikolova et al., 2006; Nikolova, 2008; Nikolova and Ivanova, 2013).

In view of symmetry, only the solution for the half plate is derived taking the origin of Cartesian coordinate at the centre of interface $I$. The plates length is denoted by $2 L$ and thickness of plates $A$ and $B$ by $2 h_{A}, 2 h_{B}$, and the Young moduli by $E_{A}$ and $E_{B}$, respectively. The thickness of structures is denoted by $2 h=2 h_{A}+2 h_{B}$ and is equal to the sum of the thickness of the plate $A$ and $B$. The uniform temperature of the plates is denoted by $\Delta T$. 


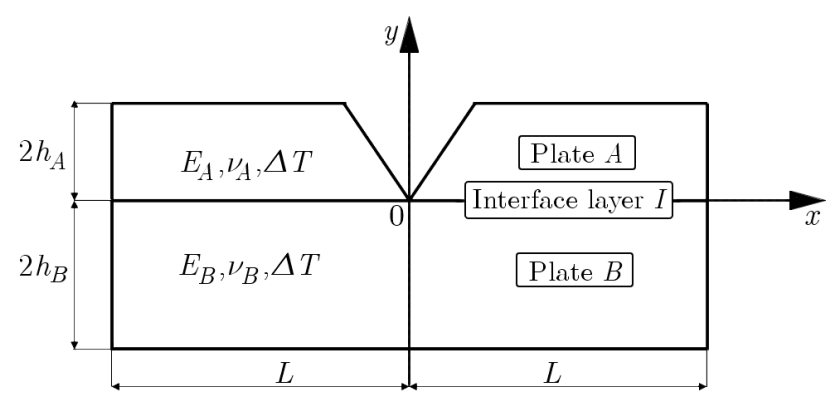

Fig. 1. Model of a Cracked two-plate structure

According to the shear lag hypothesis, the following ordinary differential equations of $1 D$ plate equilibrium can be stated

$$
\frac{d \sigma_{A}}{d x}=\frac{\tau^{I}}{2 h_{A}} \quad \frac{d \sigma_{B}}{d x}=-\frac{\tau^{I}}{2 h_{B}}
$$

where $\tau^{I}=\tau^{I}(x)$ is the interface shear stress.

The following constitutive equations for the plates and interface hold

$$
\sigma_{A}=E_{A}\left(\varepsilon_{A}-\alpha_{A} \Delta T\right) \quad \sigma_{B}=E_{B}\left(\varepsilon_{B}-\alpha_{B} \Delta T\right) \quad \tau^{I}=G^{I} w_{I}
$$

where

$$
w_{I}=\frac{u_{A}-u_{B}}{h_{A}+h_{B}}=\frac{u_{I}}{h_{A}+h_{B}} \quad \varepsilon_{A}=\frac{d u_{A}}{d x} \quad \varepsilon_{B}=\frac{d u_{B}}{d x}
$$

and $u_{A}=u_{A}(x), u_{B}=u_{B}(x)$ and $u_{I}=u_{I}(x)=u_{A}(x)-u_{B}(x)$ are the displacement fields in the plate $A$, plate $B$ and the interface. $G^{I}$ is the representative shear modulus of the interface (adhesive).

Introduce now non-dimensional variables defined as follows

$$
\begin{aligned}
& \bar{x}=\frac{x}{h} \quad \bar{u}_{i}=\frac{u_{i}}{h} \quad \bar{\sigma}_{i}=\frac{\sigma_{i}}{E_{B}} \quad \bar{\tau}^{I}=\frac{\tau^{I}}{E_{B}} \quad \bar{G}^{I}=\frac{G^{I}}{E_{B}} \\
& \bar{h}_{A}=\frac{h_{A}}{h_{A}}+h_{B} \quad \xi=\frac{h_{A}}{h_{B}} \quad \eta=\frac{E_{A}}{E_{B}} \quad i=A, B, I \quad h=h_{A}+h_{B}
\end{aligned}
$$

Then equilibrium equations (2.1) and constitutive equation (2.2) become

$$
\frac{d \bar{\sigma}_{A}}{d \bar{x}}=\frac{\bar{\tau}^{I}(1+\xi)}{2 \xi} \quad \frac{d \bar{\sigma}_{B}}{d \bar{x}}=-\frac{\bar{\tau}^{I}(1+\xi)}{2}
$$

where

$$
\bar{\sigma}_{A}=\eta\left(\bar{\varepsilon}_{A}-\alpha_{A} \Delta T\right) \quad \bar{\sigma}_{B}=\left(\bar{\varepsilon}_{B}-\alpha_{B} \Delta T\right)
$$

In subsequent derivation, the formulas will be expressed in terms of non-dimensional variables, but the dashes over the parameters will be deleted, but remembered.

\subsection{Debonding zone solution}

Let us denote $u_{I}(x)$ as $u_{I}(x)=u_{A}(x)-u_{B}(x)$. Putting the constitutive equations (2.2) in (2.1), we obtain

$$
\frac{d^{2} u_{I}}{d x^{2}}=\lambda^{2} u_{I}-\frac{d}{d x}\left[\left(\alpha_{A}-\alpha_{B}\right) \Delta T\right] \quad \lambda^{2}=\frac{G^{I}(1+\xi)(1+\xi \eta)}{2 \xi \eta}
$$


Assuming uniform temperature fields in the plates and constant thermal expansion coefficients, equation (2.6) becomes

$$
\frac{d^{2} u_{I}}{d x^{2}}=\lambda^{2} u_{I}
$$

Then equilibrium equations (2.5) can be expressed as follows

$$
\frac{d^{2} u_{A}}{d x^{2}}=\frac{\lambda^{2}}{1+\xi \eta} u_{I} \quad \frac{d^{2} u_{B}}{d x^{2}}=-\frac{\lambda^{2}}{1+\xi \eta} \xi \eta u_{I}
$$

Obviously, the substitution $u_{I}(x)=u_{A}(x)-u_{B}(x)$ has to be satisfied.

The general solution of equation (2.7) has the form

$$
u_{I}=A_{1} \cosh (\lambda x)+A_{2} \sinh (\lambda x)
$$

where are the integration constants which have to be determined.

Considering the two plate structure with a transverse crack in the first plate $A$, the following boundary and contact conditions are proposed

$$
\begin{aligned}
& u_{B}(0)=0 \Rightarrow u_{I}(0)=u_{A}(0) \\
& \sigma_{A}(0)=0 \quad \sigma_{A}(L)=\sigma_{B}(L)=0
\end{aligned}
$$

The strain-stress behavior and respective displacements can be obtained from equations (2.7) and (2.8), satisfying the above-mentioned contact and boundary conditions (2.10). The stress-strain and displacements field is presented in detail by Nikolova et al. (2006), Nikolova (2008), Nikolova and Ivanova (2013).

Now the equation for the interfacial shear stress has the following form

$$
\tau^{I}(x)=G^{I} u_{I}(x)=\frac{G^{I}(1+\xi \eta)\left(\alpha_{A}-\alpha_{B}\right) \Delta T}{\lambda} \frac{\cosh [\lambda(L-x)]}{\sinh (\lambda L)}
$$

\subsection{Length of the debonding zone}

The debond length $l_{d}$, which gives the magnitude of brittle cracking along the interface layer can be calculated from (2.11) assuming that at $\tau^{I}(x)=\tau^{c r}, u_{I}\left(l_{d}\right)=u^{c r}=\tau^{c r} / G^{I}$. Then

$$
\tau^{I}(x)=G^{I} u_{I}(x)=\frac{G^{I}(1+\xi \eta)\left(\alpha_{A}-\alpha_{B}\right) \Delta T}{\lambda} \frac{\cosh [\lambda(L-x)]}{\sinh (\lambda L)}=\tau^{c r}
$$

Using the substitution $\exp \left[\lambda\left(L-l_{d}\right)\right]=y$, we receive from $(2.12)$ the following equation for

$$
y^{2}-2 A y+1=0 \quad A=\frac{\lambda \tau^{c r} \sinh (\lambda L)}{G^{I}(1+\xi \eta)\left(\alpha_{A}-\alpha_{B}\right) \Delta T}
$$

Then two roots of (2.13) are available

$$
y_{1,2}=A \pm \sqrt{A^{2}-1}
$$

Now using the substitution $\exp \left(\lambda l_{d}\right)=y$, we obtain

$$
l_{d_{1,2}}=L-\frac{1}{\lambda} \ln \left(A \pm \sqrt{A^{2}-1}\right)
$$

Obviously, $A^{2}-1>0$.

Then we have to choose the length of the debonding zone from the condition that this length has a maximum value, i.e.

$$
l_{d}=L-\frac{1}{\lambda} \ln \left(A+\sqrt{A^{2}-1}\right)
$$




\section{Determination of the peeling stress}

\subsection{Basic equation of the dimensional peeling stress}

The basic equation for the dimensional peeling stress $p(x)$, can be obtained using the following equation of equilibrium for the thinner plate $A$ of the structure treated as an elongated thin plate (Suhir, 2006; Nikolova and Ivanova, 2013)

$$
\int_{-x_{*}}^{x} \int_{-x_{*}}^{x} p(\varsigma) d \varsigma d \varsigma+D_{1} w^{\prime \prime}(x)=\frac{h_{A}}{2} T(x)=\frac{h_{A}}{2}\left[\int_{-x_{*}}^{x} \tau^{I}(\varsigma) d \varsigma-E_{B} \tau^{c r}\left(L-l_{d}\right)\right]
$$

where

$$
D_{A}=\frac{E_{A} h_{A}^{3}}{12\left(1-\nu_{A}^{2}\right)} \quad \tau^{I}(x)=\frac{E_{B} G^{I}(1+\xi \eta)\left(\alpha_{A}-\alpha_{B}\right) \Delta T}{\lambda} \frac{\cosh [\lambda(L-x)]}{\sinh (\lambda L)}
$$

$w(x)$ is the deflection function of the plate $A$ (with respect to the thicker plate that does not experience bending deformations), $D_{A}$ is the flexural rigidity of this plate and $\tau^{I}(x)$ is the dimensional interfacial shear stress, see equations (2.4) and (2.11)

$$
T(x)=\int_{-x_{*}}^{x} \tau(\varsigma) d \varsigma-E_{B} \tau^{c r}\left(L-l_{d}\right)
$$

are the thermally induced forces acting in the cross-sections of the two-plate structure, $\tau^{c r}$ is the dimensional critical stress of the interface and $\left(L-l_{d}\right)$ is length of the intact zone, where $L$ is half the structure length. The length $l_{d}$ can be defined as $l_{d}=x_{*}=L-(1 / \lambda) \ln \left[A+\sqrt{A^{2}-1}\right]$. The origin 0 of the coordinate $x$ is in the mid-cross-section of the structure.

The peeling stress $p(x)$ can be evaluated as

$$
p(x)=K w(x)=E_{I} w(x)
$$

where $K$ is the spring constant of the elastic foundation, $w(x)$ is the deflection function and $E^{I}$ is Young's modulus of the bonding material.

We obtain the following integral equation for the peeling stress function $p(x)$

$$
\int_{-x_{*}}^{x} \int_{-x_{*}}^{x} p(\varsigma) d \varsigma d \varsigma+\frac{D_{A}}{K} p^{\prime \prime}(x)=\frac{h_{A}}{2} T(x)
$$

After differentiating this equation twice with respect to the coordinate $x$ and considering relationship (3.3), we obtain the following basic equation for the peeling stress function

$$
p^{I V}(x)+4 \beta^{4} p(x)=2 \beta^{4} h_{A} E_{B} \tau^{\prime}(x)
$$

where $\beta=\sqrt[4]{K / 4 D_{A}}$ is the parameter of the peeling stress.

In the case, when plastic strains occur in the bonding material, the following conditions must be fulfilled at the boundary, $x=x_{*}=l_{d}$, between the intact and the debonding zones

$$
\tau^{I}\left(x_{*}\right)=E_{B} \tau^{c r} \quad T\left(x_{*}\right)=-E_{B} \tau^{c r}\left(L-l_{d}\right)
$$

From (3.5) we find, by differentiation (for more details see Nikolova and Ivanova (2013))

$$
\begin{aligned}
& \int_{-x_{*}}^{x} p(\varsigma) d \varsigma+\frac{D_{A}}{K} p^{\prime \prime \prime}(x)=\frac{h_{A}}{2} E_{B} \tau^{I}(x) \\
& \int_{-x_{*}}^{x_{*}} \int_{-x_{*}}^{x} p(\varsigma) d \varsigma d \varsigma=0 \quad \int_{-x_{*}}^{x_{*}} p(\varsigma) d \varsigma=0
\end{aligned}
$$


With consideration of conditions $(3.8)_{2}$, relationships (3.5) and (3.8) $)_{1}$ result in the following boundary conditions for the peeling stress function $p(x)$

$$
\begin{aligned}
& p^{\prime \prime}\left(x_{*}\right)=-\frac{h_{A} K l_{e} \tau^{c r}}{4 D_{A}}=-2 \beta^{4} h_{A}\left(L-l_{d}\right) E_{B} \tau^{c r} \\
& p^{\prime \prime \prime}\left(x_{*}\right)=\frac{h_{A} K \tau^{c r}}{2 D_{A}}=2 \beta^{4} h_{A} E_{B} \tau^{c r}
\end{aligned}
$$

The peeling stress in the debonding zone should be zero as it follows from equation (3.6). The dimensional shear stress is equal to the dimensional critical stress between the intact and the debonding zones.

\subsection{Solution to the peeling stress equation}

Equation (3.6) has form of an equation of a beam lying on a continuous elastic foundation. We seek a solution to this equation in form

$$
p(x)=C_{0} V_{0}(\beta x)+C_{1} V_{1}(\beta x)+C_{2} V_{2}(\beta x)+C_{3} V_{3}(\beta x)+B \frac{\sinh \left[\lambda\left(L-x_{*}\right)\right]}{\sinh (\lambda x)}
$$

These functions $p(x)$ are odd functions, i.e. $p(-x)=-p(x)$. They have their maximum value (zero derivative) at the origin, and are symmetric with respect to the mid-cross-section of the assembly.

The final form of the solution to equation (3.6) is the following

$$
p(x)=C_{0} V_{0}(\beta x)+C_{2} V_{2}(\beta x)+B \frac{\sinh \left[\lambda\left(L-x_{*}\right)\right]}{\sinh (\lambda x)}
$$

where the functions $V_{i}(\beta x), i=0,2$ are expressed as follows

$$
V_{0}(\beta x)=\cosh (\beta x) \cos (\beta x) \quad V_{2}(\beta x)=\sinh (\beta x) \sin (\beta x)
$$

The first two terms in (3.11) provide the general solution to the homogeneous equation, which corresponds to non-homogeneous equation (3.6), and the third term is the particular solution to this equation. Introducing this term into equation (3.6), we obtain

$$
(3.13) B=\frac{2 G^{I} E_{B} h_{A} \beta^{4}\left(\alpha_{A}-\alpha_{B}\right) \Delta T(1+\xi \eta)}{4 \beta^{4}+\lambda^{4}}
$$

Using boundary conditions (3.9), we obtain the following algebraic equations for the constants $C_{0}$ and $C_{2}$ of integration

$$
\begin{aligned}
& -2 \beta^{3}\left[\sin \left(\beta x_{*}\right) \cosh \left(\beta x_{*}\right)+\sinh \left(\beta x_{*}\right) \cos \left(\beta x_{*}\right)\right] C_{0} \\
& \quad-2 \beta^{3}\left[\sin \left(\beta x_{*}\right) \cosh \left(\beta x_{*}\right)+\sinh \left(\beta x_{*}\right) \cos \left(\beta x_{*}\right)\right] C_{2}=2 \beta^{4} h_{A} E_{B} \tau^{c r} \\
& -\beta\left[\sin \left(\beta x_{*}\right) \cosh \left(\beta x_{*}\right)-\sinh \left(\beta x_{*}\right) \cos \left(\beta x_{*}\right)\right] C_{0} \\
& \quad+\beta\left[\sin \left(\beta x_{*}\right) \cosh \left(\beta x_{*}\right)+\sinh \left(\beta x_{*}\right) \cos \left(\beta x_{*}\right)\right] C_{2}=-2 \beta^{4} h_{A}\left(L-l_{d}\right) E_{B} \tau^{c r}
\end{aligned}
$$

Algebraic equations (3.14) have the following solutions

$$
\begin{aligned}
& C_{0}=\frac{\beta h_{A} E_{B}\left[\sin \left(\beta x_{*}\right) \cosh \left(\beta x_{*}\right)\left(1-2 \beta^{2} L+2 \beta^{2} l_{d}\right)+\sinh \left(\beta x_{*}\right) \cos \left(\beta x_{*}\right)\left(1+2 \beta^{2} L-2 \beta^{2} l_{d}\right)\right] \tau^{c r}}{\cos \left(2 \beta x_{*}\right)-\cosh \left(2 \beta x_{*}\right)} \\
& C_{2}=\frac{\beta h_{A} E_{B}\left[\sin \left(\beta x_{*}\right) \cos \left(\beta x_{*}\right)\left(1+2 \beta^{2} L-2 \beta^{2} l_{d}\right)-\sinh \left(\beta x_{*}\right) \cos \left(\beta x_{*}\right)\left(1-2 \beta^{2} L+2 \beta^{2} l_{d}\right)\right] \tau^{c r}}{\cos \left(2 \beta x_{*}\right)-\cosh \left(2 \beta x_{*}\right)}
\end{aligned}
$$


Note that for long enough elastic zones, solution (3.11) can be simplified as follows

$$
\begin{aligned}
p(x) & =\frac{\beta h_{A} E_{B}\left[\sin \left(\beta x_{*}\right) \cosh \left(\beta x_{*}\right)\left(1-2 \beta^{2} L+2 \beta^{2} l_{d}\right)\right] \tau^{c r}}{\cos \left(2 \beta x_{*}\right)-\cosh \left(2 \beta x_{*}\right)} \cosh (\beta x) \cos (\beta x) \\
& +\frac{\beta h_{A} E_{B}\left[\sinh \left(\beta x_{*}\right) \cos \left(\beta x_{*}\right)\left(1+2 \beta^{2} L-2 \beta^{2} l_{d}\right)\right] \tau^{c r}}{\cos \left(2 \beta x_{*}\right)-\cosh \left(2 \beta x_{*}\right)} \cosh (\beta x) \cos (\beta x) \\
& +\frac{\beta h_{A} E_{B}\left[\sin \left(\beta x_{*}\right) \cos \left(\beta x_{*}\right)\left(1+2 \beta^{2} L-2 \beta^{2} l_{d}\right)\right] \tau^{c r}}{\cos \left(2 \beta x_{*}\right)-\cosh \left(2 \beta x_{*}\right)} \sinh (\beta x) \sin (\beta x) \\
& -\frac{\beta h_{A} E_{B}\left[\sinh \left(\beta x_{*}\right) \cos \left(\beta x_{*}\right)\left(1-2 \beta^{2} L+2 \beta^{2} l_{d}\right)\right] \tau^{c r}}{\cos \left(2 \beta x_{*}\right)-\cosh \left(2 \beta x_{*}\right)} \sinh (\beta x) \sin (\beta x) \\
& +\frac{2 G^{I} E_{B} h_{A} \beta^{4}\left(\alpha_{A}-\alpha_{B}\right) \Delta T(1+\xi \eta)}{4 \beta^{4}+\lambda^{4}} \frac{\sinh \left[\lambda\left(L-x_{*}\right)\right]}{\sinh (\lambda x)}
\end{aligned}
$$

\section{Numerical example}

Let us consider two elastic plates $A$ (made from $\mathrm{ZrO}_{2}$ ) and $B$ (made from $\mathrm{Ti}_{6} \mathrm{Al}_{4} \mathrm{~V}$ ) bonded by the interface with finite lengths $2 L=120 \mathrm{~mm}$ and variable thickness of the first plate $h_{A}$ $2 h_{A}=[1.6 \mathrm{~mm}, 2 \mathrm{~mm}], 2 h_{B}=2 \mathrm{~mm}, h=h_{A}+h_{B}=[1.8 \mathrm{~mm}, 2 \mathrm{~mm}], \xi=h_{A} / h_{B}=[0.8,1]$ under monotonic temperature loading $\Delta T$. The following material characteristics are taken:

- $\mathrm{ZrO}_{2}$ - Young's modulus $E_{A}=132.2 \mathrm{GPa}$, coefficient of thermal expansion $\alpha_{A}=$ $=13.3 \cdot 10^{-6} \mathrm{~K}^{-1}$,

- $\mathrm{Ti}_{6} \mathrm{Al}_{4} \mathrm{~V}-$ Young's modulus $E_{B}=122.7 \mathrm{GPa}$, coefficient of thermal expansion $\alpha_{B}=$ $=10.291 \cdot 10^{-6} \mathrm{~K}^{-1}$,

- interface layer - Young's modulus $E^{I}=2.1 \mathrm{GPa}$, shear modulus $G^{I}=800 \mathrm{MPa}$ and the critical shear stress $\tau^{I}=\tau^{c r}=18 \mathrm{MPa}$.

In Table 1, the variable parameters of the problem considered are presented.

Table 1. Variable parameter

\begin{tabular}{|l|c|}
\hline \multicolumn{1}{|c|}{ Name } & Variable \\
\hline \hline Thickness of the first plate $A$ & $h_{A}$ \\
\hline Geometric parameter of the structure & $\xi=h_{A} / h_{B}$ \\
\hline Non-dimensional parameter & $\lambda=\sqrt{G^{I}(1+\xi)(1+\xi \eta) / 2 \xi \eta}$ \\
\hline Flexural rigidity of the plate $A$ & $D_{A}=E_{A} h_{A}^{3} /\left[12\left(1-\nu_{A}^{2}\right)\right]$ \\
\hline Parameter of the peeling stress & $\beta=\sqrt[4]{K / 4 D_{A}}$ \\
\hline Temperature monotonic loading & $\Delta T$ \\
\hline
\end{tabular}

The behavior of the dimensional interfacial shear and the peeling stresses on $x / h$, the dimensional peeling stress $p(x)$ for three different temperature of the monotonic loading $\Delta T$ and as well as the behavior of the respective peeling stresses $p(x)$ for three different values of the parameter $\xi=h_{A} / h_{B}$ are illustrated at the given bellow figures (Figs. 2, 3, and 4).

The analytically calculated shear and peeling stresses are plotted in Fig. 2 for a temperature $\Delta T=350 \mathrm{~K}$. As shown in this figure, the interfacial shear stress is equal to the critical shear stress at the point between the intact and debonding zones. Thereafter, the shear stress decreases sharply from its maximum value at the interface end (the dependence of the interfacial shear stress for different temperatures is presented in detail in Nikolova et al. (2006), Nikolova (2008)).

Contrary to the shear stress, the interfacial peeling stress attains a maximum value at the end of the interface. From the beginning of the intact zone, the peeling stress has a negative value and then increases to its maximum value (less than the critical shear stress value). 


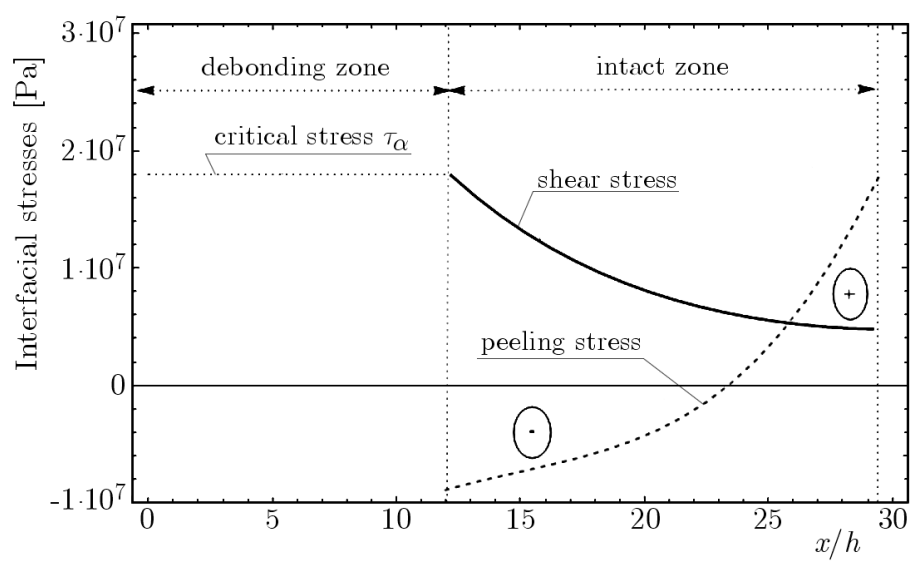

Fig. 2. Calculated interfacial stresses

Figure 3 shows the behavior of the peeling stresses $p(x)$ for $\xi=h_{A} / h_{B}=1$ and three different values of temperature loading $\Delta T$. The peeling stress again attains a maximum value at the end of the interface layer and is negative in the middle of the intact zone.

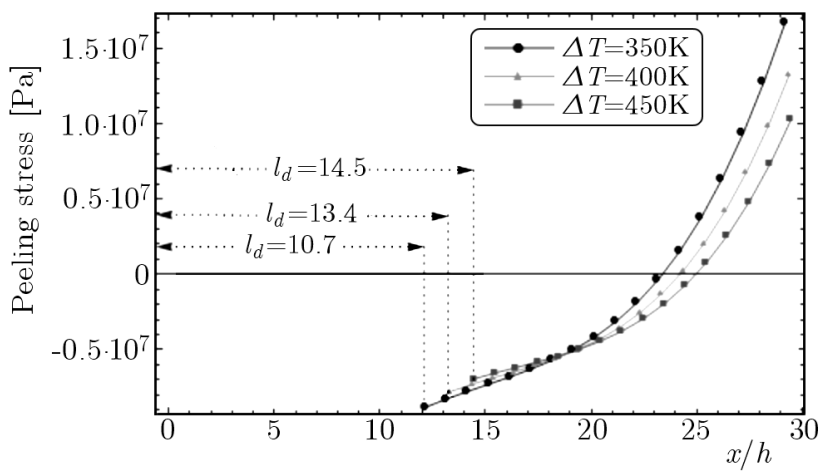

Fig. 3. Dependence of $p(x)$ on $x / h$ for different temperature loadings $\Delta T$

The maximum peeling stress decreases with a rise of the temperature loading $\Delta T$.

For a given value $h_{B}=0.001 \mathrm{~m}$, three different thicknesses $h_{A}$ and $\Delta T=350 \mathrm{~K}$, the interfacial peeling stresses are plotted as a function of the axial distance in Fig. 4.

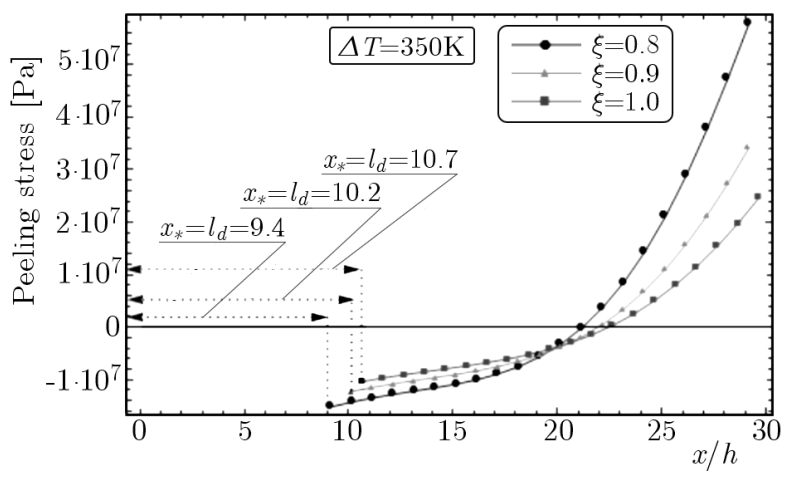

Fig. 4. Dependence of the peeling stresses $p(x)$ on $x / h$ for three different values of the parameter $\xi$

The thickness of material $A$ is varied in a range $h_{A}=0.0008 \mathrm{~m}-0.001 \mathrm{~m}$. The peeling stress $p(x)$ decreases with a increase in the parameter $\xi$ and attains its maximum value at the interface end, strongly depending on the thickness of plate (material) $A$. The maximum peeling stress increases with a decrease in the plate $A$ thickness $h_{A}$, while the area for the peeling stress region decreases with the increasing of the thickness $h_{A}$. 
The dependence of the interfacial peeling stress is sensitive to material properties and geometry of the bi-material structure.

When the thickness of the first plate is very small, the peeling stress increases extremely rapidly and exceeds the critical shear stress value, then in the structure the full debonding of the interface can be observed.

\section{Conclusions}

The shear lag method, classical plate theory formulation and elastic foundation theory have been used to investigate the interfacial shear and peeling stresses in a pre-cracked bi-material structure subjected to monotonic thermal loading.

The following results can be summarized:

(1) The lengths of the debonding and intact zones are calculated by means of the shear lag analysis associated with constitutive equations.

(2) The peeling stress is sensitive to material properties, geometry of the pre-cracked bi-material structure and the applied thermal loading. Therefore, the thermally induced interfacial peeling stresses in different ceramic-metal composites are preferable to be with low maximum values.

(3) Interfacial shear and peeling stresses due to thermal and elastic mismatch in layered structures are one of the major reasons of mechanical and thermal failure and delamination in multilayered structures.

(4) The obtained from the present analysis results can be used for the assessment of thermally induced stresses in different pre-cracked ceramic-metal composites during production of new ideally designed ceramic-metal composites with optimal properties of combining high temperature resistance and hardness (ceramic), and the ability to undergo plastic deformation (metal).

Acknowledgement

The study has been financially supported by the National Fund "Scientific Research" of Bulgaria, Project DFNI E02/10121214.

\section{References}

1. Beuth J.L., 1992, Cracking of thin films in thin bonded films in residual tension, International Journal of Solids and Structures, 29, 13, 1567-1675

2. Beuth J.L., Klingbeil N.W., 1996, Cracking of thin films bonded to elastic-plastic substrates, Journal of the Mechanics and Physics of Solids, 44, 9, 1411-1428

3. Bleeck O., Munz D., Schaller W., Yang Y.Y., 1998, Effect of a graded interlayer on the stress intensity factor of cracks in a joint under thermal loading, Engineering Fracture Mechanics, 60, 615-623

4. Cox L.H., 1952, The elasticity and strength of paper and other fibrous materials, British Journal of Applied Physics, 3, 72-79

5. Dowling P.J., Burgan B.A., 1990, Shear lag in steel and composite structures, [In:] Composite Steel Structures. Advances, Design and Construction, edited by R. Narayanan, Spon Press, London, $1-23$

6. Hutchinson J.W., Suo Z., 1991, Mixed mode cracking in layered materials, Advances in Applied Mechanics, 29, 63-191 
7. NAIRN J.A., 1988a, Fracture mechanics of unidirectional composites using the shear-lag model I: theory, Journal of Composite Materials, 22, 6, 561-588

8. NAIRN J.A., 1988b, Fracture mechanics of unidirectional composites using the shear-lag model II: experiment, Journal of Composite Materials, 22, 6, 589-600

9. Nikolova G., 2008, Thermo-mechanical behaviour of thin multilayered structures, Ph.D. Thesis, Institute of Mechanics - BAS, Sofia, Bulgaria

10. Nikolova G., Ivanova J., 2013, Interfacial shear and peeling stresses in a two-plate structure subjected to monotonically increasing thermal loading, Journal of Theoretical and Applied Mechanics, $\mathbf{5 1 , ~ 4 , ~ 9 3 7 - 9 4 7 ~}$

11. Nikolova G., Ivanova J., Mroz Z., 2006, Modelling of thermally induced progressive delamination in a two-plate structure, Journal of Theoretical and Applied Mechanics, Bulgaria, 36, 4, 71-92

12. Sorensen B.F., Jorgensen S.O., Horsewell A., 1998, Thermally induced delamination of multilayer, Acta Matterial, 46, 2603-2615

13. SuHir E., 2006, Interfacial thermal stresses in a bi-material assembly with a low-yield-stress bonding layer, Journal of Physics D: Modeling and Simulation in Materials Science and Engineering, 14, 1421-1432

14. Zhang S., 2000, Thermal stress intensities at an interface crack between two elastic layers, International Journal of Fracture, 106, 277-29 